\title{
A note on nonlinear singular integral operators depending on two parameters
}

\author{
Gumrah Uysal ${ }^{1}$ and Ertan Ibikli ${ }^{2}$ \\ ${ }^{1}$ Department of Mathematics, Karabuk University, Karabuk,Turkey \\ ${ }^{2}$ Department of Mathematics, Ankara University, Ankara, Turkey
}

Received: 30 July 2015, Revised: 5 August 2015, Accepted: 21 October 2015

Published online: 18 January 2016.

\begin{abstract}
In this paper we present some theorems concerning pointwise convergence of nonlinear singular integral operators of the form:

$$
T_{\lambda}(f ; x)=\int_{D} K_{\lambda}(t-x ; f(t)) d t, x \in D, \lambda \in \Lambda
$$

where $D=\langle a, b\rangle$ is open, half-open or closed arbitrary bounded interval in $\mathbb{R}$ or $D=\mathbb{R}$ and $\Lambda \neq \emptyset$ be the set of indices, at a common point of continuity and Lebesgue point of the functions $f \in L_{1, \varphi}(D)$ and $\varphi \in L_{1}(D)$. Here, $L_{1, \varphi}(D)$ is the space of all measurable functions for which $\left|\frac{f}{\varphi}\right|$ is integrable on $D$. Also we investigate the rate of pointwise convergence at Lebesgue points.
\end{abstract}

Keywords: Continuity point, Lebesgue point, rate of convergence, Lipschitz condition, nonlinear integral operators.

\section{Introduction}

In [15], Taberski, who emphasized the significance of singular integrals in Fourier analysis in his studies, analyzed the pointwise approximation of functions $f \in L_{1}\langle-\pi, \pi\rangle$ and their derivatives by a family of convolution type linear singular integral operators depending on two parameters of the form:

$$
L_{\lambda}(f ; x)=\int_{-\pi}^{\pi} f(t) K_{\lambda}(t-x) d t, x \in\langle-\pi, \pi\rangle, \lambda \in \Lambda \subset \mathbb{R}_{0}^{+}
$$

where the symbol $\langle-\pi, \pi\rangle$ stands for closed, half-open or open interval and $K_{\lambda}(t)$ is the kernel satisfying suitable assumptions. Subsequently, the pointwise convergence of the operators of type (1) was examined by Gadjiev [5] and Rydzewska [12] at generalized Lebesgue points and $\mu$-generalized Lebesgue points of functions $f \in L_{1}\langle-\pi, \pi\rangle$, respectively. Then, Karsli and Ibikli [6] generalized the results of [5], [15] and [12] by handling the operators of type (1) in the space $L_{1}\langle a, b\rangle$. Almali [1] studied the pointwise convergence of non-convolution type integral operators to non-integrable functions at Lebesgue points. For further studies on linear singular operators in different settings, the reader may see also e.g. [13]-[19]. 
Later on, Musielak [10] improved the notion of singularity to include the case of nonlinear integral operators of the form:

$$
T_{w} f(y)=\int_{G} K_{w}(x-y ; f(x)) d x, y \in G, w \in \Lambda
$$

where $G$ be a locally compact Abelian group equipped with Haar measure and $\Lambda \neq \emptyset$ be an index set with any topology, via replacing the linearity property of the operators by an assumption of Lipschitz condition for $K_{w}$ with respect to second variable. The studies, which were published until that time, showed that the singularity of the operators was related to their linearity [3]. Afterwards, Swiderski and Wachnicki [14] investigated the pointwise convergence of the operators of type (2) at $p$-Lebesgue points of the functions $f \in L_{p}(-\pi, \pi)$.

Today, approximation via nonlinear integral operators is extensively used in many branches of science such as medicine and engineering. Especially, effect of nonlinear integral operators in sampling theory must be emphasized here [3]. Further, signal and image processing are two main research fields around sampling theory. In view of this situation, one would prefer to investigate the convergence of nonlinear integral operators rather than the convergence of linear integral operators. For some advanced studies, auhors refer to [2] and [3].

The current manuscript is a continuation and further generalzation of [7] and deals with the pointwise convergence of nonlinear singular integral operators of the form:

$$
T_{\lambda}(f ; x)=\int_{D} K_{\lambda}(t-x ; f(t)) d t, x \in D, \lambda \in \Lambda
$$

where $D=\langle a, b\rangle$ is open, half-open or closed arbitrary bounded interval in $\mathbb{R}$ or $D=\mathbb{R}, \Lambda \neq \emptyset$ be the set of indices, at a common continuity point and Lebesgue point of the functions $f \in L_{1, \varphi}(D)$ and $\varphi \in L_{1}(D)$. Here, $L_{1, \varphi}(D)$ is the space of all measurable functions for which $\left|\frac{f}{\varphi}\right|$ is integrable on $D$.

In Section 2, we introduce fundamental notions. In Section 3, we prove the existence of the operators of type (3). In Section 4 , we give two theorems concerning the pointwise convergence of $T_{\lambda}(f ; x)$. In Section 5 , we establish the rate of pointwise convergence of the operators of type (3).

\section{Preliminaries}

Definition 1. (Class $\left.A_{\varphi}\right)$ Suppose that the function $K_{\lambda}: \mathbb{R} \times \mathbb{R} \rightarrow \mathbb{R}$ is integrable for each $\lambda \in \Lambda$ and satisfies the property expressed as $K_{\lambda}(\vartheta, 0)=0$ for every $\vartheta \in \mathbb{R}$ and for each $\lambda \in \Lambda$. Further, let $w(t)=\sup _{x \in D}\left[\frac{\varphi(t+x)}{\varphi(x)}\right]$ for every $t \in \mathbb{R}$, where $\varphi: \mathbb{R} \rightarrow \mathbb{R}^{+}$is a weight function and the following conditions are satisfied:

(a) There exists an integrable function $L_{\lambda}: \mathbb{R} \rightarrow \mathbb{R}$ such that the following inequality:

$$
\left|K_{\lambda}(t, u)-K_{\lambda}(t, v)\right| \leq L_{\lambda}(t)|u-v|
$$

holds for every $t, u, v \in \mathbb{R}$ and for each $\lambda \in \Lambda$.

(b) $\left\|w L_{\lambda}\right\|_{L_{1}(\mathbb{R})} \leq M<\infty, \quad \forall \lambda \in \Lambda$.

(c) $\lim _{\lambda \rightarrow \lambda_{0}}\left[\sup _{t \in \mathbb{R} \backslash \Omega} L_{\lambda}(t)\right]=0, \forall \Omega \in N(0)$, where $N(0)$ stands for all neighborhoods of the number 0 in $\mathbb{R}$. 
(d) $\lim _{\lambda \rightarrow \lambda_{0}}\left[\int_{\mathbb{R} \backslash \Omega} L_{\lambda}(t) d t\right]=0, \forall \Omega \in N(0)$.

(e) For a given $\delta_{0}>0, L_{\lambda}(t)$ is non-decreasing function with respect to $t$ on $\left\langle-\delta_{0}, 0\right]$ and non-increasing function with respect to $t$ on $\left[0, \delta_{0}\right\rangle$.

(f) $\lim _{\lambda \rightarrow \lambda_{0}}\left|\int_{\mathbb{R}} K_{\lambda}(t, u) d t-u\right|=0, \forall u \in \mathbb{R}$.

Remark. For the Lipschitz condition $(a)$, authors refer to $[9,10]$. Besides, one may take $\varphi(t)=1$ when $f$ is integrable.

Class $A_{\varphi}$ is a kernel by condition (a). Also, throughout this article we suppose that $K_{\lambda}$ belongs to Class $A_{\varphi}$ and $f \notin L_{1}(D)$.

\section{Existence of operators}

Main results in this work are based on the following theorem.

Theorem 1. If $f \in L_{1, \varphi}(D)$, then the operator $T_{\lambda}(f ; x) \in L_{1, \varphi}(D)$ and

$$
\left\|T_{\lambda}(f ; x)\right\|_{L_{1, \varphi}(D)} \leq\left\|w L_{\lambda}\right\|_{L_{1}(\mathbb{R})}\|f\|_{L_{1, \varphi}(D)}
$$

for all $\lambda \in \Lambda$.

Proof. Let $D=\langle a, b\rangle$. Further, let us define a new function $g$ by

$$
g(t):=\left\{\begin{array}{c}
f(t), \text { if } \quad t \in\langle a, b\rangle, \\
0, \text { if } t \in \mathbb{R} \backslash\langle a, b\rangle .
\end{array}\right.
$$

Using conditian (a) and Fubini's Theorem (see, for example, [4]), we can write

$$
\begin{aligned}
\left\|T_{\lambda}(f ; x)\right\|_{L_{1, \varphi}\langle a, b\rangle} & =\int_{a}^{b} \frac{1}{\varphi(x)} \int_{a}^{b} K_{\lambda}(t-x ; f(t)) d t \mid d x \\
& \leq \int_{a}^{b} \frac{1}{\varphi(x)} \int_{a}^{b}\left|K_{\lambda}(t-x ; f(t))-K_{\lambda}(t-x ; 0)\right| d t d x \\
& \leq \int_{a}^{b} \frac{1}{\varphi(x)} \int_{-\infty}^{\infty}\left|g(t+x) \frac{\varphi(t+x)}{\varphi(t+x)} L_{\lambda}(t)\right| d t d x \\
& =\int_{a}^{b} \frac{1}{\varphi(x)} \int_{-\infty}^{\infty}\left|\varphi(t+x) \frac{g(t+x)}{\varphi(t+x)} L_{\lambda}(t)\right| d t d x \\
& =\int_{a}^{b} \frac{1}{\varphi(x)}\left[\int_{-\infty}^{\infty} \varphi(t+x)\left|\frac{g(t+x)}{\varphi(t+x)}\right| L_{\lambda}(t) d t\right] d x \\
& =\int_{-\infty}^{\infty} L_{\lambda}(t)\left[\int_{a}^{b} \frac{\varphi(t+x)}{\varphi(x)}\left|\frac{g(t+x)}{\varphi(t+x)}\right| d x\right] d t \\
& \left.\leq\left\|w L_{\lambda}\right\|_{L_{1}(\mathbb{R})}\|f\|_{L_{1, \varphi}\langle a, b\rangle \cdot}\right]
\end{aligned}
$$


Here the norm of $f \in L_{1, \varphi}\langle a, b\rangle$ is given by the following equality (see e.g., [8]):

$$
\|f\|_{L_{1, \varphi}\langle a, b\rangle}=\int_{a}^{b}\left|\frac{f(x)}{\varphi(x)}\right| d x .
$$

The assertion may be proven with the above method for the case $D=\mathbb{R}$. Thus the proof is completed.

\section{Convergence at characteristic points}

Theorem 2. Suppose that $\varphi(t)$ and $L_{\lambda}(t-x)$ are almost everywhere differentiable functions on $\mathbb{R}$ with respect to $t$ such that the following inequality:

$$
\frac{d}{d t} \varphi(t) \frac{d}{d t} L_{\lambda}(t-x)>0, \text { for any fixed } x \in \mathbb{R}
$$

holds. If $x_{0} \in \mathbb{R}$ is a common continuity point of the functions $f \in L_{1, \varphi}(\mathbb{R})$ and $\varphi \in L_{1}(\mathbb{R})$, then

$$
\lim _{(x, \lambda) \rightarrow\left(x_{0}, \lambda_{0}\right)} T_{\lambda}(f ; x)=f\left(x_{0}\right)
$$

on any set $Z$ on which the function

$$
\int_{x_{0}-\delta}^{x_{0}+\delta} \varphi(t) L_{\lambda}(t-x) d t
$$

is bounded as $(x, \lambda)$ tends to $\left(x_{0}, \lambda_{0}\right)$.

Proof. Let $0<x_{0}-x<\frac{\delta}{2}$ for a given $\delta>0$. Set $I=\left|T_{\lambda}(f ; x)-f\left(x_{0}\right)\right|$. From Theorem 2 in [7], we have

$$
\begin{aligned}
I & =\left|\int_{-\infty}^{\infty} K_{\lambda}(t-x ; f(t)) d t-f\left(x_{0}\right)\right| \\
& =\mid \int_{-\infty}^{\infty} K_{\lambda}(t-x ; f(t)) d t-\int_{-\infty}^{\infty} K_{\lambda}\left(t-x ; \frac{f\left(x_{0}\right)}{\varphi\left(x_{0}\right)} \varphi(t)\right) d t \\
& +\int_{-\infty}^{\infty} K_{\lambda}\left(t-x ; \frac{f\left(x_{0}\right)}{\varphi\left(x_{0}\right)} \varphi(t)\right) d t-f\left(x_{0}\right) \mid
\end{aligned}
$$

Using condition ( $a$ ) of class $A_{\varphi}$, we have

$$
\begin{aligned}
I & \leq \int_{-\infty}^{\infty}\left|\frac{f(t)}{\varphi(t)}-\frac{f\left(x_{0}\right)}{\varphi\left(x_{0}\right)}\right| \varphi(t) L_{\lambda}(t-x) d t \\
& +\left|\int_{-\infty}^{\infty} K_{\lambda}\left(t-x ; \frac{f\left(x_{0}\right)}{\varphi\left(x_{0}\right)} \varphi(t)\right) d t-f\left(x_{0}\right)\right| \\
& =I_{1}+I_{2} .
\end{aligned}
$$


From Theorem 2 in [7], $I_{2} \rightarrow 0$ as $(x, \lambda)$ tends to $\left(x_{0}, \lambda_{0}\right)$. Clearly, $I_{1}$ can be written in the form:

$$
\begin{aligned}
I_{1} & =\left\{\int_{\mathbb{R} \backslash\left\langle x_{0}-\delta, x_{0}+\delta\right\rangle}+\int_{\left\langle x_{0}-\delta, x_{0}+\delta\right\rangle}\right\}\left|\frac{f(t)}{\varphi(t)}-\frac{f\left(x_{0}\right)}{\varphi\left(x_{0}\right)}\right| \varphi(t) L_{\lambda}(t-x) d t \\
& =I_{11}+I_{12} .
\end{aligned}
$$

By (4) and condition (e) of class $A_{\varphi}$, we may write

$$
I_{11} \leq\left\{\varphi\left(x_{0}-\delta\right)+\varphi\left(x_{0}+\delta\right)\right\}\left\{\sup _{|\xi|>\frac{\delta}{2}} L_{\lambda}(\xi)\|f\|_{L_{1, \varphi}(\mathbb{R})}+\left|\frac{f\left(x_{0}\right)}{\varphi\left(x_{0}\right)}\right| \int_{|\xi|>\frac{\delta}{2}} L_{\lambda}(\xi) d \xi\right\}
$$

Therefore, by conditions $(c)$ and $(d)$ of class $A_{\varphi}, I_{11} \rightarrow 0$ as $(x, \lambda)$ tends to $\left(x_{0}, \lambda_{0}\right)$. Since the function $\frac{f(t)}{\varphi(t)}$ is continuous at $t=x_{0}$, we have

$$
I_{12} \leq \varepsilon \int_{x_{0}-\delta}^{x_{0}+\delta} \varphi(t) L_{\lambda}(t-x) d t
$$

The remaining part of the proof is clear by the hypothesis (5). Hence

$$
\lim _{(x, \lambda) \rightarrow\left(x_{0}, \lambda_{0}\right)} T_{\lambda}(f ; x)=f\left(x_{0}\right)
$$

Also, using similar proof method, same conclusion is obtained for the case $0<x-x_{0}<\frac{\delta}{2}$ for a given $\delta>0$. Thus the proof is completed.

Theorem 3. Suppose that $\varphi(t)$ and $L_{\lambda}(t-x)$ are almost everywhere differentiable functions on $\mathbb{R}$ with respect to $t$ such that the following inequality:

$$
\frac{d}{d t} \varphi(t) \frac{d}{d t} L_{\lambda}(t-x)>0, \text { for any fixed } x \in\langle a, b\rangle
$$

holds. If $x_{0} \in\langle a, b\rangle$ is a common Lebesgue point of the functions $f \in L_{1, \varphi}\langle a, b\rangle$ and $\varphi \in L_{1}\langle a, b\rangle$, then

$$
\lim _{(x, \lambda) \rightarrow\left(x_{0}, \lambda_{0}\right)} T_{\lambda}(f ; x)=f\left(x_{0}\right)
$$

on any set $Z$ on which the function

$$
\int_{x_{0}-\delta}^{x_{0}+\delta} \varphi(t) L_{\lambda}(t-x) d t+2 L_{\lambda}(0) \varphi(x)\left|x-x_{0}\right|
$$

is bounded as $(x, \lambda)$ tends to $\left(x_{0}, \lambda_{0}\right)$.

Proof. Let

$$
x_{0}+\delta<b, x_{0}-\delta>a \text { and } 0<x_{0}-x<\frac{\delta}{2}
$$


for a given $\delta>0$. Set $I=\left|T_{\lambda}(f ; x)-f\left(x_{0}\right)\right|$. From Theorem 3 in [7], we may write

$$
\begin{aligned}
I & =\left|\int_{a}^{b} K_{\lambda}(t-x ; f(t)) d t-f\left(x_{0}\right)\right| \\
& =\mid \int_{a}^{b} K_{\lambda}(t-x ; f(t)) d t-\int_{a}^{b} K_{\lambda}\left(t-x ; \frac{f\left(x_{0}\right)}{\varphi\left(x_{0}\right)} \varphi(t)\right) d t \\
& +\int_{a}^{b} K_{\lambda}\left(t-x ; \frac{f\left(x_{0}\right)}{\varphi\left(x_{0}\right)} \varphi(t)\right) d t-f\left(x_{0}\right) \mid .
\end{aligned}
$$

Using condition $(a)$ of class $A_{\varphi}$, the following inequality:

$$
\begin{aligned}
I & \leq \int_{a}^{b}\left|\frac{f(t)}{\varphi(t)}-\frac{f\left(x_{0}\right)}{\varphi\left(x_{0}\right)}\right| \varphi(t) L_{\lambda}(t-x) d t \\
& +\left|\int_{a}^{b} K_{\lambda}\left(t-x ; \frac{f\left(x_{0}\right)}{\varphi\left(x_{0}\right)} \varphi(t)\right) d t-f\left(x_{0}\right)\right| \\
& =I_{1}+I_{2}
\end{aligned}
$$

is obtained. From Theorem 3 in [7], $I_{2} \rightarrow 0$ as $(x, \lambda)$ tends to $\left(x_{0}, \lambda_{0}\right)$. Now, $I_{1}$ can be written in the form:

$$
\begin{aligned}
I_{1} & =\left\{\int_{\left\langle x_{0}-\delta, x_{0}+\delta\right\rangle}+\int_{\langle a, b\rangle \backslash\left\langle x_{0}-\delta, x_{0}+\delta\right\rangle}\right\}\left|\frac{f(t)}{\varphi(t)}-\frac{f\left(x_{0}\right)}{\varphi\left(x_{0}\right)}\right| \varphi(t) L_{\lambda}(t-x) d t \\
& =I_{11}+I_{12} .
\end{aligned}
$$

Next, we consider the integral $I_{11}$. Splitting $I_{11}$ into two parts yields the following:

$$
\begin{aligned}
I_{11} & =\left\{\int_{x_{0}-\delta}^{x_{0}}+\int_{x_{0}}^{x_{0}+\delta}\right\}\left|\frac{f(t)}{\varphi(t)}-\frac{f\left(x_{0}\right)}{\varphi\left(x_{0}\right)}\right| \varphi(t) L_{\lambda}(t-x) d t \\
& =I_{111}+I_{112} .
\end{aligned}
$$

By the definition of Lebesgue point (see, e.g., [11] and [14]), for every $\varepsilon>0$ there exists a corresponding number $\delta_{0}>0$ such that

$$
\int_{x_{0}-h}^{x_{0}}\left|\frac{f(t)}{\varphi(t)}-\frac{f\left(x_{0}\right)}{\varphi\left(x_{0}\right)}\right| d t<\varepsilon h
$$

holds for all $0<h \leq \delta<\delta_{0}$. Now, we define the function $F(t)$ by

$$
F(t)=\int_{t}^{x_{0}}\left|\frac{f(y)}{\varphi(y)}-\frac{f\left(x_{0}\right)}{\varphi\left(x_{0}\right)}\right| d y .
$$


Therefore, we have

$$
d F(t)=-\left|\frac{f(t)}{\varphi(t)}-\frac{f\left(x_{0}\right)}{\varphi\left(x_{0}\right)}\right| d t .
$$

From (8) and (9) for all $t$ satisfying the condition $0<x_{0}-t \leq \delta<\delta_{0}$ we have

$$
F(t) \leq \varepsilon\left(x_{0}-t\right)
$$

By virtue of (9) and (10) we get

$$
\begin{aligned}
I_{111} & =\int_{x_{0}-\delta}^{x_{0}}\left|\frac{f(t)}{\varphi(t)}-\frac{f\left(x_{0}\right)}{\varphi\left(x_{0}\right)}\right| L_{\lambda}(t-x) \varphi(t) d t \\
& =\int_{x_{0}-\delta}^{x_{0}} L_{\lambda}(t-x) \varphi(t) d[-F(t)] .
\end{aligned}
$$

Using integration by parts and applying (11), we have the following inequality:

$$
\begin{aligned}
\left|I_{111}\right| & \leq \varepsilon \delta L_{\lambda}\left(x_{0}-\delta-x\right) \varphi\left(x_{0}-\delta\right)+\varepsilon \int_{x_{0}-\delta}^{x_{0}}\left(x_{0}-t\right)\left|d L_{\lambda}(t-x) \varphi(t)\right| \\
& =\varepsilon \delta L_{\lambda}\left(x_{0}-\delta-x\right) \varphi\left(x_{0}-\delta\right)+\varepsilon \int_{x_{0}-x-\delta}^{x_{0}-x}\left(x_{0}-x-t\right)\left|d L_{\lambda}(t) \varphi(t+x)\right| .
\end{aligned}
$$

Now, we define the following variation function:

$$
E(t)=\left\{\begin{array}{cc}
\vee^{t} & L_{\lambda}(u) \varphi(u+x), x_{0}-x-\delta<t \leq x_{0}-x, \\
x_{0}-x-\delta & t=x_{0}-x-\delta .
\end{array}\right.
$$

Using above variation we have

$$
\left|I_{111}\right| \leq \varepsilon \delta L_{\lambda}\left(x_{0}-\delta-x\right) \varphi\left(x_{0}-\delta\right)+\varepsilon \int_{x_{0}-x-\delta}^{x_{0}-x}\left(x_{0}-x-t\right)|d E(t)|
$$

Now, if we use hypothesis (6) and integration by parts, then we have the following expression:

$$
\begin{aligned}
\left|I_{111}\right| \leq-\varepsilon \int_{x_{0}-x-\delta}^{x_{0}-x}\left(E(t)+L_{\lambda}\left(x_{0}-\delta-x\right) \varphi\left(x_{0}-\delta\right)\right)\left\{x_{0}-x-t\right\}_{t}^{\prime} d t \\
=\varepsilon \int_{x_{0}-x-\delta}^{x_{0}-x}\left(E(t)+L_{\lambda}\left(x_{0}-\delta-x\right) \varphi\left(x_{0}-\delta\right)\right) d t .
\end{aligned}
$$


We evaluate the above integral using condition $(e)$ of class $A_{\varphi}$. Therefore, we obtain

$$
\left|I_{111}\right| \leq \varepsilon\left\{\int_{x_{0}-\delta}^{x_{0}} L_{\lambda}(t-x) \varphi(t) d t+2 L_{\lambda}(0) \varphi(x)\left|x_{0}-x\right|\right\}
$$

Using same strategy, we can estimate the integral $I_{112}$ as

$$
\left|I_{112}\right| \leq \varepsilon \int_{x_{0}}^{x_{0}+\delta} L_{\lambda}(t-x) \varphi(t) d t
$$

Combining $\left|I_{111}\right|$ and $\left|I_{112}\right|$, we obtain

$$
I_{11} \leq \varepsilon\left\{\int_{x_{0}-\delta}^{x_{0}+\delta} L_{\lambda}(t-x) \varphi(t) d t+2 L_{\lambda}(0) \varphi(x)\left|x_{0}-x\right|\right\}
$$

In view of hypothesis (7), if the points $(x, \lambda) \in Z$ are sufficiently near to $\left(x_{0}, \lambda_{0}\right)$, then $I_{11}$ tends to zero. The remaining part of the proof is analogous to proof of the preceding theorem. Therefore, $I_{12} \rightarrow 0$ as $(x, \lambda)$ tends to $\left(x_{0}, \lambda_{0}\right)$. Also, using similar proof method, the same conclusion is obtained for the case $0<x-x_{0}<\frac{\delta}{2}$ for a given $\delta>0$. Hence, the proof is completed.

Theorem 4. Suppose that $\varphi(t)$ and $L_{\lambda}(t-x)$ are almost everywhere differentiable functions on $\mathbb{R}$ with respect to $t$ such that the following inequality:

$$
\frac{d}{d t} \varphi(t) \frac{d}{d t} L_{\lambda}(t-x)>0, \text { for any fixed } x \in \mathbb{R}
$$

holds. If $x_{0} \in \mathbb{R}$ is a common Lebesgue point of the functions $f \in L_{1, \varphi}(\mathbb{R})$ and $\varphi \in L_{1}(\mathbb{R})$, then

$$
\lim _{(x, \lambda) \rightarrow\left(x_{0}, \lambda_{0}\right)} T_{\lambda}(f ; x)=f\left(x_{0}\right)
$$

on any set $Z$ on which the function

$$
\int_{x_{0}-\delta}^{x_{0}+\delta} \varphi(t) L_{\lambda}(t-x) d t+2 L_{\lambda}(0) \varphi(x)\left|x-x_{0}\right|
$$

is bounded as $(x, \lambda)$ tends to $\left(x_{0}, \lambda_{0}\right)$.

Proof. Set $I=\left|T_{\lambda}(f ; x)-f\left(x_{0}\right)\right|$. Making similar calculations as in the proof of Theorem 3, we have

$$
I \leq\left\{\varphi\left(x_{0}-\delta\right)+\varphi\left(x_{0}+\delta\right)\right\}\left\{\sup _{|\xi|>\frac{\delta}{2}} L_{\lambda}(\xi)\|f\|_{L_{1, \varphi}(\mathbb{R})}+\left|\frac{f\left(x_{0}\right)}{\varphi\left(x_{0}\right)}\right| \int_{|\xi|>\frac{\delta}{2}} L_{\lambda}(\xi) d \xi\right\}
$$




$$
\begin{aligned}
& +\varepsilon \int_{x_{0}-\delta}^{x_{0}+\delta} L_{\lambda}(t-x) \varphi(t) d t+2 \varepsilon L_{\lambda}(0) \varphi(x)\left|x_{0}-x\right| \\
& +\left|\int_{-\infty}^{\infty} K_{\lambda}\left(t-x ; \frac{f\left(x_{0}\right)}{\varphi\left(x_{0}\right)} \varphi(t)\right) d t-f\left(x_{0}\right)\right| .
\end{aligned}
$$

Using conditions $(b)$ and $(c)$ of class $A_{\varphi}$ and from Theorem 4 in [7], we obtain $I \rightarrow 0$ as $(x, \lambda) \rightarrow\left(x_{0}, \lambda_{0}\right)$. Hence the proof is completed.

\section{Rate of convergence}

In this section, two theorems concerning rate of pointwise convergence will be given.

Theorem 5. Suppose that the hypothesis of Theorem 3 is satisfied. Let

$$
\Delta(x, \lambda, \delta)=\int_{x_{0}-\delta}^{x_{0}+\delta} L_{\lambda}(t-x) \varphi(t) d t+2 L_{\lambda}(0) \varphi(x)\left|x_{0}-x\right|
$$

where $0<\delta<\delta_{0}$, and the following conditions are satisfied:

(i) $\Delta(x, \lambda, \delta) \rightarrow 0$ as $(x, \lambda) \rightarrow\left(x_{0}, \lambda_{0}\right)$ for some $\delta>0$.

(ii) For every $\xi>0$,

$$
L_{\lambda}(\xi)=o(\Delta(x, \lambda, \delta))
$$

as $(x, \lambda) \rightarrow\left(x_{0}, \lambda_{0}\right)$
$\left|\int_{a}^{b} K_{\lambda}\left(t-x ; \frac{f\left(x_{0}\right)}{\varphi\left(x_{0}\right)} \varphi(t)\right) d t-f\left(x_{0}\right)\right|=o(\Delta(x, \lambda, \delta))$.

Then at each common Lebesque point of functions $f \in L_{1, \varphi}\langle a, b\rangle$ and $\varphi \in L_{1}\langle a, b\rangle$ we have as $(x, \lambda) \rightarrow\left(x_{0}, \lambda_{0}\right)$

$$
\left|T_{\lambda}(f ; x)-f\left(x_{0}\right)\right|=o(\Delta(x, \lambda, \delta))
$$

Proof. Under the hypothesis of Theorem 3, we write

$$
\begin{aligned}
\left|T_{\lambda}(f ; x)-f(x)\right| \leq & \left\{\varphi\left(x_{0}-\delta\right)+\varphi\left(x_{0}+\delta\right)\right\} \\
& \times \sup _{|\xi|>\frac{\delta}{2}} L_{\lambda}(\xi)\left\{\|f\|_{L_{1, \varphi}\langle a, b\rangle}+\left|\frac{f\left(x_{0}\right)}{\varphi\left(x_{0}\right)}\right|(b-a)\right\} \\
& +\varepsilon \int_{x_{0}-\delta}^{x_{0}+\delta} L_{\lambda}(t-x) \varphi(t) d t+2 \varepsilon L_{\lambda}(0) \varphi(x)\left|x_{0}-x\right| \\
& +\left|\int_{a}^{b} K_{\lambda}\left(t-x ; \frac{f\left(x_{0}\right)}{\varphi\left(x_{0}\right)} \varphi(t)\right) d t-f\left(x_{0}\right)\right| .
\end{aligned}
$$

From (i)-(iii), we have the desired result i.e.:

$$
\left|T_{\lambda}(f ; x)-f\left(x_{0}\right)\right|=o(\Delta(x, \lambda, \delta)) .
$$


Theorem 6. Suppose that the hypothesis of Theorem 4 is satisfied. Let

$$
\Delta(x, \lambda, \delta)=\int_{x_{0}-\delta}^{x_{0}+\delta} L_{\lambda}(t-x) \varphi(t) d t+2 L_{\lambda}(0) \varphi(x)\left|x_{0}-x\right|
$$

where $0<\delta<\delta_{0}$, and the following conditions are satisfied:

(i) $\Delta(x, y, \lambda, \delta) \rightarrow 0$ as $(x, \lambda) \rightarrow\left(x_{0}, \lambda_{0}\right)$ for some $\delta>0$.

(ii) For every $\xi>0$,

$$
L_{\lambda}(\xi)=o(\Delta(x, \lambda, \delta))
$$

as $(x, \lambda) \rightarrow\left(x_{0}, \lambda_{0}\right)$.

(iii) For every $\xi>0$,

$$
\lim _{\lambda \rightarrow \lambda_{0}} \int_{|t|>\xi} L_{\lambda}(t) d t=o(\Delta(x, \lambda, \delta))
$$

as $(x, \lambda) \rightarrow\left(x_{0}, \lambda_{0}\right)$.
(iv) $\left|\int_{-\infty}^{\infty} K_{\lambda}\left(t-x ; \frac{f\left(x_{0}\right)}{\varphi\left(x_{0}\right)} \varphi(t)\right) d t-f\left(x_{0}\right)\right|=o(\Delta(x, \lambda, \delta))$.

Then at each common Lebesque point of functions $f \in L_{1, \varphi}(\mathbb{R})$ and $\varphi \in L_{1}(\mathbb{R})$ we have as $(x, \lambda) \rightarrow\left(x_{0}, \lambda_{0}\right)$

$$
\left|T_{\lambda}(f ; x)-f\left(x_{0}\right)\right|=o(\Delta(x, \lambda, \delta))
$$

Proof. Under the hypothesis of Theorem 4, we write

$$
\begin{aligned}
&\left|T_{\lambda}(f ; x)-f\left(x_{0}\right)\right| \leq \sup _{|\xi|>\frac{\delta}{2}} L_{\lambda}(\xi)\left(\varphi\left(x_{0}-\delta\right)+\varphi\left(x_{0}+\delta\right)\right)\|f\|_{L_{1, \varphi}(\mathbb{R})} \\
&+\left(\varphi\left(x_{0}-\delta\right)+\varphi\left(x_{0}+\delta\right)\right)\left|\frac{f\left(x_{0}\right)}{\varphi\left(x_{0}\right)}\right| \int_{|\xi|>\frac{\delta}{2}} L_{\lambda}(\xi) d \xi \\
&+ \int_{x_{0}-\delta}^{x_{0}+\delta} L_{\lambda}(t-x) \varphi(t) d t+2 \varepsilon L_{\lambda}(0) \varphi(x)\left|x_{0}-x\right| \\
&+\left|\int_{-\infty}^{\infty} K_{\lambda}\left(t-x ; \frac{f\left(x_{0}\right)}{\varphi\left(x_{0}\right)} \varphi(t)\right) d t-f\left(x_{0}\right)\right| .
\end{aligned}
$$

From (i)-(iv), we have the desired result i.e.:

$$
\left|T_{\lambda}(f ; x)-f\left(x_{0}\right)\right|=o(\Delta(x, \lambda, \delta))
$$

\section{References}

[1] S. E Almali, Convergence and the order of convergence of family of nonconvolution type integral operators at characteristics points, Ph. D. Thesis, Ankara University, Graduate School of Applied Science, Ankara, 2002. 
[2] C. Bardaro, J. Musielak, G. Vinti, Approximation by nonlinear singular integral operators in some modular function spaces, Ann. Polon. Math, 63(1996), no. 2, 173-182.

[3] C. Bardaro, J. Musielak, G. Vinti, Nonlinear integral operators and applications, DeGruyter Series in Nonlinear Analysis and Applications, 9(2003), xii + 201 pp.

[4] P. L. Butzer and R. J. Nessel, Fourier Analysis and Approximation, vol. I. Academic Press, Newyork, London, 1971.

[5] A. D. Gadjiev, The order of convergence of singular integrals which depend on two parameters, Special Problems of Functional Analysis and their Appl. to the Theory of Diff. Eq. and the Theory of Func., Izdat. Akad. Nauk Azerbădažan. SSR., (1968), 40-44.

[6] H. Karsli and E. Ibikli, On convergence of convolution type singular integral operators depending on two parameters, Fasc. Math., 38(2007), 25-39.

[7] H. Karsli and E. Ibikli, On convergence and rate of convergence of nonlinear singular integral operators, Proc. Jangjeon Math. Soc., 9(2006), no. 2, 137-150.

[8] R. G. Mamedov, On the order of convergence of m-singular integrals at generalized Lebesgue points and in the space $L p(-\infty, \infty)$, Izv. Akad. Nauk SSSR Ser. Mat 27(1963), no.2, 287-304.

[9] J. Musielak, Approximation by nonlinear singular integral operators in generalized Orlicz spaces, Comment. Math. Prace Mat. 31 (1991), 79-88.

[10] J. Musielak, On some approximation problems in modular spaces, In Constructive Function Theory 1981, (Proc. Int. Conf., Varna, June 1-5, 1981), Publ. House Bulgarian Acad. Sci., Sofia 1983, 455-461.

[11] W. Rudin, Real and Complex Analysis, Mc-Graw Hill Book Co., London, 1987.

[12] B. Rydzewska, Approximation des fonctions par des intégrales singulières ordinaires, Fasc. Math., vol. 7, (1973), 71-81.

[13] E. M. Stein, Singular Integrals and Differentiability of Functions, Princeton Univ. Press, New Jersey, 1970.

[14] T. Swiderski and E. Wachnicki, Nonlinear singular integrals depending on two parameters, Commentationes Math., 40(2000), 181-189.

[15] R. Taberski, Singular integrals depending on two parameters, Rocznicki Polskiego towarzystwa matematycznego, Seria I. Prace matematyczne, 7(1962), 173-179.

[16] R. Taberski, On double integrals and Fourier Series, Ann. Polon. Math. 15(1964), 97-115.

[17] G. Uysal, M. M. Yilmaz, E. Ibikli, A study on pointwise approximation by double singular integral operators, J. Inequal. Appl., 2015:94, 2015.

[18] G. Uysal and M. M. Yilmaz, Some theorems on the approximation of non-integrable functions via singular integral operators, Proc. Jangjeon Math. Soc., 18(2015), no. 2, 241-251.

[19] M. M. Yilmaz, G. Uysal, E. Ibikli, A note on rate of convergence of double singular integral operators, Adv. Difference Equ., 2014:287, 2014. 